\title{
Traffic Light Control System using Genetic Algorithm
}

\author{
Nuka D. Nwiabu \\ Department of Computer Science \\ Rivers State University Port Harcourt, \\ Nigeria
}

\author{
Emem E. Udoudom \\ Department of Computer Science \\ Rivers State University Port Harcourt, \\ Nigeria
}

\begin{abstract}
Genetic Algorithm (GA) technology in the traffic control system to provide intelligent green interval responses based on dynamic traffic load inputs, thereby overcoming the inefficiencies of the conventional fixed traffic controllers.

In this paper, the authors explore the use of genetic algorithm and implementing the technology to improve the performance of traffic light and Road control in a four-way, two-lane traffic. The algorithm resolves the limitations of traditional fixed-time control for passing vehicles. It employs a dynamic system to control the traffic light system that monitors two sets of parameters: the vehicle and upstream and downstream lane queues behind a red light and the number of vehicles that passes through a green light. The algorithm dynamically optimizes the red and green times to control the flow of the vehicles. Performance comparisons between the Dynamic traffic controller and a fixed-time controller reveal that the genetic algorithm controller performs significantly better. The authors compare the performance of their algorithm with the unimproved one for different simulated data. Results show that, the algorithm increases the traffic efficiency and decreases the waiting delay by 30 minute compared with the unimproved one.
\end{abstract}

\section{General Terms}

Traffic Control System

\section{Keywords}

Genetic Algorithm, Traffic Control System, Traffic Light, Optimization.

\section{INTRODUCTION}

Genetic Algorithms are search algorithms based on the mechanics of natural selection and genetics. They combine the concept of survival of the fittest among string structures with a structured yet randomized information exchange to form a search algorithm with some of the innovative flair of human search [1]. As early as 1962, John Holland's work on adaptive systems laid the foundation for later developments. He develop and popularize the field with his work in the early 1970s at the University of Michigan focus on Genetic algorithms (GA) which are a type of optimization algorithm, meaning they are used to find the optimal solution(s) to a given computational problem that maximizes or minimizes a particular function [2]. John Holland's devised a method, or schema theorem, for evaluating each generation of genetic algorithms. Genetic Algorithm is a heuristic function for optimization, where the extreme of the function (i.e., minimal or maximal) cannot be established analytically. A population of potential solutions is refined iteratively by employing a strategy inspired by Darwinist evolution or natural selection. Genetic Algorithms promote "survival of the fittest". This type of heuristic has been applied in many different fields, including construction of neural networks and finance [3].Additionally, in the GA technique, the crossover operator mixes and matches attributes between two chromosomes through random processes. Two random selections are applied successively, namely, the random selection of two chromosomes from the chromosome population and then that of an arbitrary gene position within the chromosomes (referred to as the crossover point). The number of crossovers can be restricted to one per pair, two per pair, or $\mathrm{N}$ per pair. These and other variations of the basic algorithm have been discussed extensively by [4]. This work explores the use of genetic algorithm and implementing the technology to improve the performance of traffic light and Road control in a four-way, two-lane traffic. The algorithm resolves the limitations of traditional fixed-time control for passing vehicles. It employs a dynamic system to control the traffic light system that monitors two sets of parameters: the vehicle and upstream and downstream lane queues behind a red light and the number of vehicles that passes through a green light. The algorithm dynamically optimizes the red and green times to control the flow of the vehicles. Performance comparisons between the Dynamic traffic controller and a fixed-time controller reveal that the genetic algorithm controller performs significantly better. The remainder of this paper provides; 2 . Brief overview of related works. 3. The methodologies. 4. Genetic Algorithm and Traffic System. Traffic Control System Using Genetic Algorithm. 5. Results and Discussions. 6. Conclusion and further work.

\section{LITERATURE REVIEW}

Genetic Algorithm is an adaptive and efficient heuristics that is able to solve optimization problems. This is a stochastic search technique to look for optimal solution. Most genetic algorithms are used in research and science related works to look for optimal solutions [5].The features inherent in genetic algorithm play a critical role in making them the best choice for practical applications, namely optimization, computer aided design, scheduling, economics and game theory. It is also selected because it does not require the presence of supervisor or observer [6].However, genetic algorithms, without prior training, continuously allow permanent renewal of decisions in generating solutions. Instead of trying to optimize a single solution, they work with a population of candidate solutions that are encoded as chromosomes. Within these chromosomes are separate genes that represent the independent variables for the problem at hand. There are a number of specific attributes of genetic algorithms that give them an edge over other traditional optimization techniques. These are: A genetic algorithm works from a population, not a single point, and hence it is likely to be trapped at a local optimum. Traffic Control System, Derivative freeness, i.e., a genetic algorithm does not need the objective function's derivative to do its work[7]. In [8] further the efficiency of genetic algorithm has been mathematically established, Solution to problems using genetic approach involves encoding and evaluation. The parameters of interest are converted into codes and combined together to form a chromosome. Further the solution is evaluation for a fitness 
function in an iterative manner. Shows genetic algorithm process where an initial population of solution of a given problem is generated and the value of fitness function (which has to be minimized / maximized) for each solution is calculated. the solution with better fitness function has a higher fitness function has a higher probability of survival .considering the main procedure of finding the best fit using genetic algorithm

\section{Reproduction \\ II. Crossover}

III. Mutation

In addition [9] Dept. of Computer Sci., West Univ. of Timisoara, Timisoara, Romania , Use of Genetic Algorithms in Numerical Weather Prediction, Numerical weather prediction models have been used for the past decades to forecast and research atmospheric circulation, at first at large scales with many limitations. With the advance of technology and computer science, meteorology became a quantitative science and forecast models became more accessible to scientists, forecasters and interested persons, weather prediction is focused more on particular forecasts rather than general, playing an important role in society with many activities becoming more dependent on weather modeling development. In [10] develop Static and adaptive distributed data replication using genetic algorithms. Fast dissemination and access of information in large distributed systems, such as the Internet, has become a norm of our daily life. However, undesired long delays experienced by end-users, especially during the peak hours, continue to be common problems

\section{METHODOLOGIES}

To achieve the aim of this work, the following methodologies were exploited;

\subsection{Experimental Research}

Experimental research method is the straightforward experiment, involving the standard practice of manipulating quantitative, independent variables to generate statistically analyzable data. Generally, the system of scientific measurements is interval or ratio based. When we talk about 'scientific research methods', this is what most people immediately think of, because it passes all of the definitions of 'true science'. The researcher is accepting or refuting the null hypothesis. The results generated are analyzable and are used to test hypotheses, with statistics giving a clear and unambiguous picture [11]

This enables researchers to compare the two groups and determine the impact of the intervention following processes were considered: survey, questionnaires, and interview

\subsection{Agile Method}

Agile software design methodology is a combination of iterative and incremental process models with focus on process adaptability and customer satisfaction by rapid delivery of working software product. Agile Methods break the product into small incremental builds. These builds are provided in iterations [12]. Each iteration typically lasts from about one to three weeks. Every iteration involves cross functional teams working simultaneously on various areas like
III. Design
IV. Coding
V. Unit Testing and
VI. Acceptance Testing.

At the end of the iteration, a working product is displayed to the customer and important stakeholders.

\section{TRAFFIC CONTROL SYSTEM'S ARCHITECTURE}

The proposed system uses genetic algorithm to simulate traffic in an intersection of four (4) ways lane roads for the purpose of research and study by the Traffic Engineers. The specification or Requirement for performing simulations on the proposed system is given in Table 4.1. The requirement was collected through observation, interview from the user and referring to the database for real data. The requirement specifications typically includes the components necessary for implementing a given software process. It also includes some key information about the type and nature of application domain (such as the use of genetic domains e.g. the use of genetic algorithm domain). It must be emphasized that these requirements may change depending on the Application domain.

The initial requirements specifications will hence include:

1. A formal definition of the primary components required for implementing the system (e.g. the use of genetic algorithm).

2. A description of the data attribute structure of the functional objects/or attributes in the software system (state some key data structures here) See below and use as appropriate.

Table 1 show the requirement specification data obtained from Rivers State ministry of transportation, port Harcourt Nigeria. The proposed system uses genetic algorithm for traffic light system (artificial system) to evolve a set of system parameters from which context is built and predictions are made. The design includes the use of real time data from the roads. It basically consists of an input layer, optimization layer and decision layer. The Architecture of Predictive/decision making System is shown in Figure 1. The system consists of input layer, optimization layer and decision layer. The input layer receives as input the attribute range specifications.

The optimization layer uses the genetic algorithm to evolve and then find the set of parameters that gives least cost. By least cost it is meant, the least error as defined by an objective function.

A decision layer that takes decisions (e.g. setting of sensor) based on the condition of input against a reference value.

The optimization layer uses the genetic algorithm to evolve and then find the set of parameters that gives least cost. By least cost it is meant, the least error as defined by an objective function. A decision layer that takes decisions (e.g. setting of sensor detector) based on the condition of input against a reference value.

\section{Planning \\ II. Requirements Analysis}


Table 1:Sample Input/output Specifications for the genetic algorithm for traffic light control system/decision making system, source Rivers State Ministry of Transport.

\begin{tabular}{|c|c|c|c|c|}
\hline \multirow{2}{*}{$\begin{array}{l}\text { Traffic } \\
\text { mode }\end{array}$} & \multirow[t]{2}{*}{ Sensor Counter } & \multirow{2}{*}{$\begin{array}{c}\text { Light signal (NORTH/SOUTH )/EAST } \\
\text { WEST Detector }\end{array}$} & \multicolumn{2}{|c|}{ Vehicle composition } \\
\hline & & & $<-49 \%$ & $>=50 \%$ \\
\hline \multirow{7}{*}{$\begin{array}{c}\text { Bicycle } \\
\text { Truck } \\
\text { Auto } \\
\text { Car } \\
\text { Mini bus } \\
\text { Van }\end{array}$} & Peak period & Nature of the road density & 43 & 89 \\
\hline & peak period & Nature of the road density & 45 & 70 \\
\hline & Off Peak period & Nature of the road density & 17 & 37 \\
\hline & Off peak & Nature of the road density & 1.4 & 20 \\
\hline & Off peak & Nature of the road density & 1.2 & 20 \\
\hline & Peak peak & Nature of the road density & 57 & 90 \\
\hline & Off peak & Nature of the road density & 34 & 40 \\
\hline
\end{tabular}

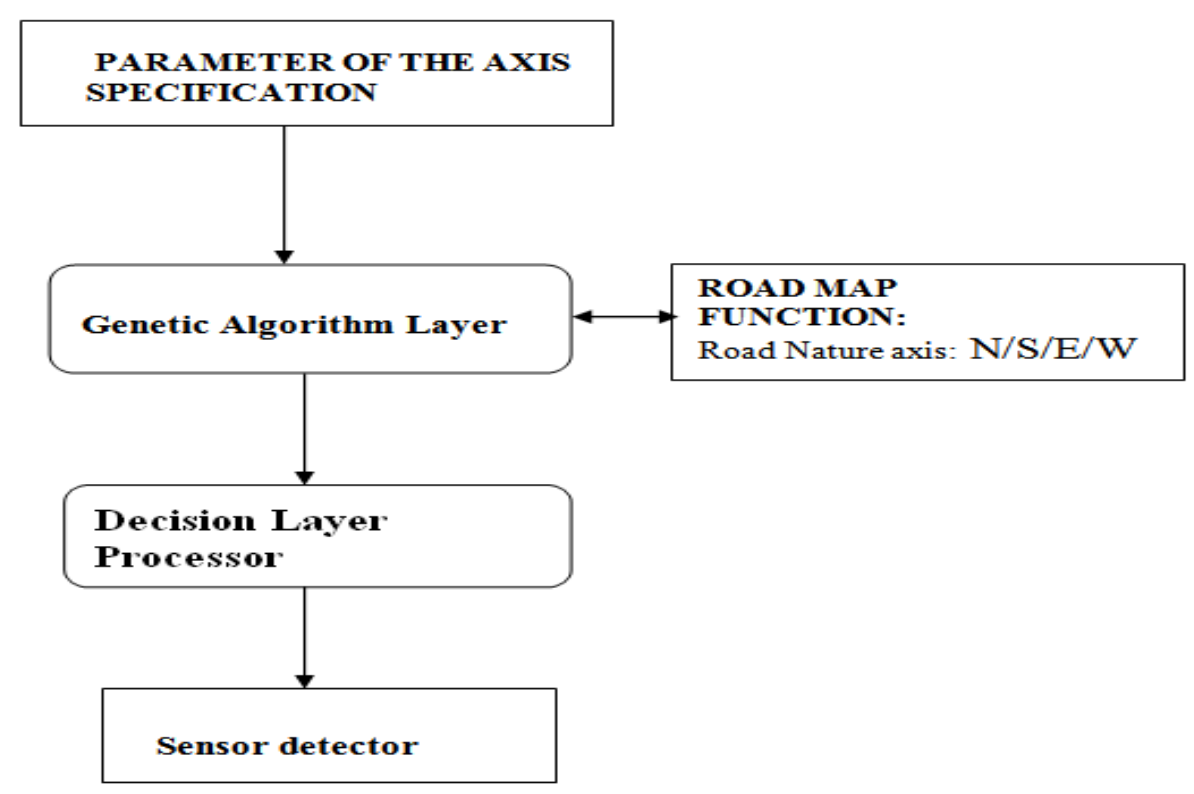

Figure 1: Architecture of Proposed Traffic Light Control System using Genetic Algorithm

The system consists of three layers, which are input module, optimization module and decision module. Input module is where to input the attribute range specifications like the speed of car, peak period/off peak period assessment setting etc. Optimization layer uses the AI technique (genetic algorithm) to simulate and then find the set of sensor setting parameter that gives least cost, the least error as defined by an objective function which will be used by the decision module for optimization of road efficiency. Decision module executes the operation based on the condition of input data against a reference value that will be used to simulate the best fit if there is proper measure, efficient of sensor in the road traffic light control system. These hands-on methods are working for St. Pete, for now, but growth is steady. And though interstates in the area are being expanded, surface roads can't be widened. And growing outward isn't even an option, thanks to the glistening ocean surrounding the city on three sides.
Genetic algorithm is use to optimize and fins the best fit nature of the road is betting on alternative transit like cycling ,cars and busing to reduce the number of cars on the road. The city is outfitting a few major surface roads with advanced sensors and processors that will adjust signals automatically, from real fix time road nature system.

The genetic algorithm traffic sensing technology, like that in most cities, is still largely limited to induction loops embedded in asphalt. These loops can detect a single vehicle waiting on a small side road, triggering a green signal across a major artery. But induction loops can't distinguish a single car from an angry traffic jam. Newer sensors can not only detect density, but distinguish between cars, buses, bicycles, and pedestrians, letting algorithms dish out green lights with a bigger picture in mind. so genetic algorithm uses the best fit to search and view the nature of the road by so doing it choose the best path to indicate green for movement. The system is further explained using a component diagram as shown in fig. 2. 


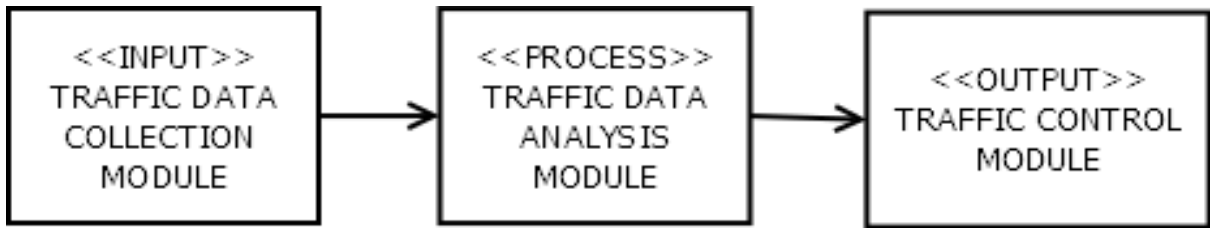

Figure 2: Proposed Systems Component Architecture

The component of the system is made up of three parts:

i. Input Module (Traffic Data Collection Module): The input module collects the data on the traffic on the road. This data include the size of the number of cars on the road.

ii. Process Module (Traffic Data Analysis Module): This module used the Genetic Algorithm to analyze the data so as to determine the pattern of traffic on the road iii. Output Module (Traffic Control Module): The traffic control module will use the analyzed data collected to control the traffic on the road taking into consideration the size of the traffic on the road. The snapshots of the various modules that make up proposed Traffic Management Simulation System along with a description of their functions are shown in figure 3,4 and 5 .

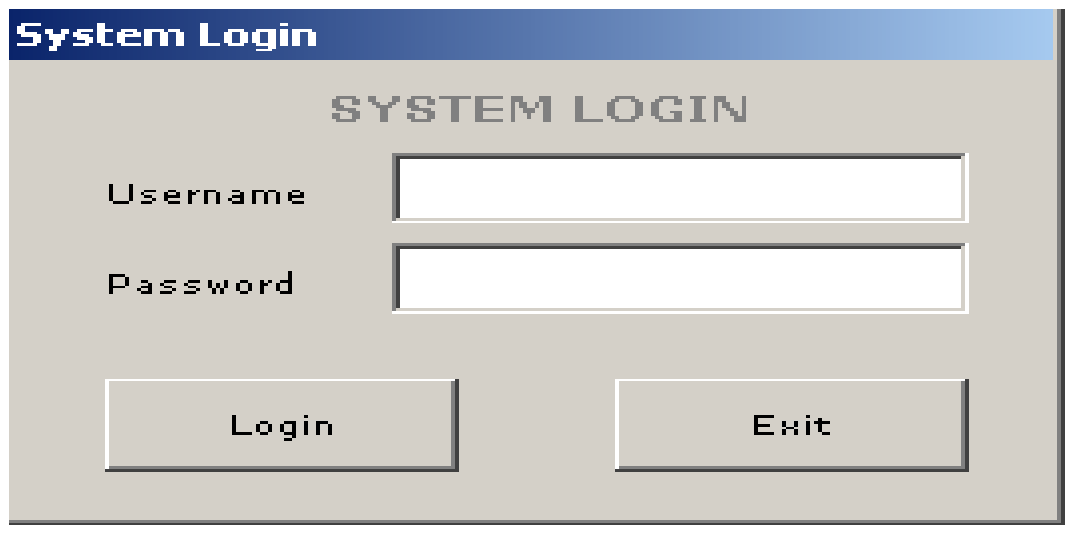

Figure 3: System Login Form

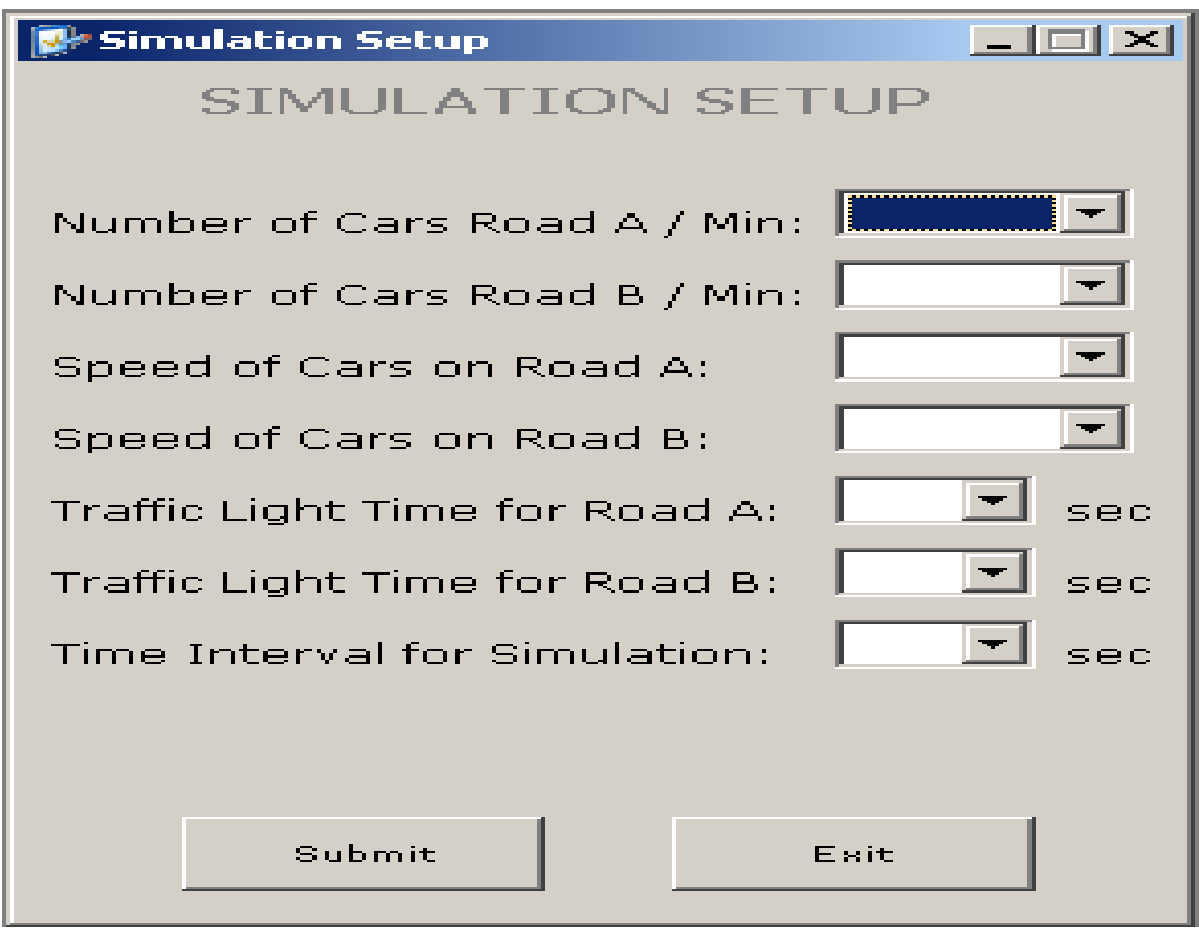

Figure 4: Simulation Setup Form

The System Login Form (see figure 3) allows the user submit login details to gain access into the system. The Simulation Setup Form of fig.4 allows is used to setup a simulation exercise.
The simulation window form (see figure 5) displays the graphic simulation of traffic on the intersection of the Roads $\mathrm{A}$ and $\mathrm{B}$. 


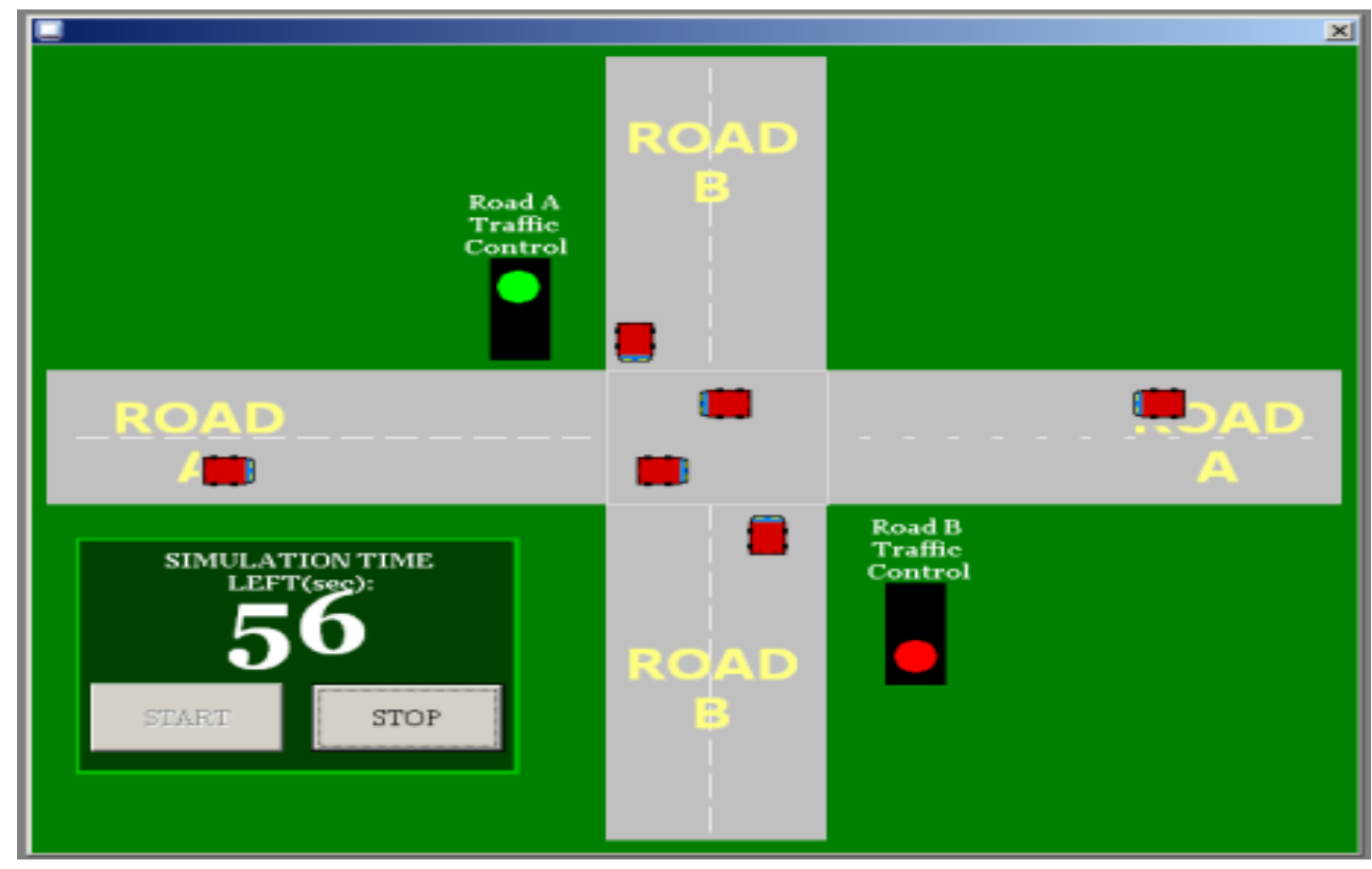

Figure 5: Snapshot of the Simulation Window

\section{RESULTS AND DISCUSSION}

Figure 6 displays information on the result of the simulation exercise.

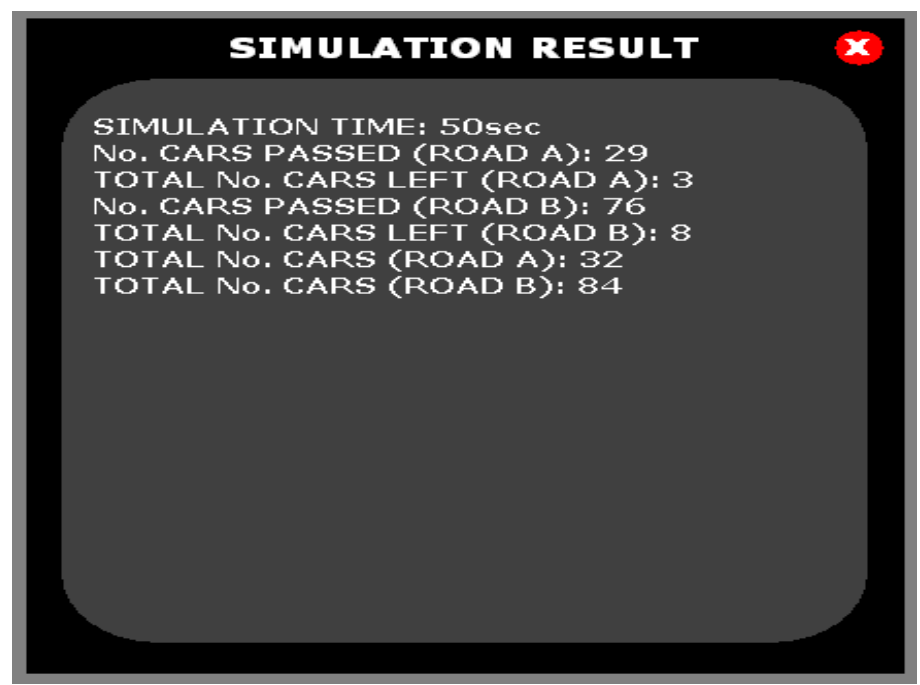

Fig. 6 Snapshot of Simulation Exercise

The tables below show simulation results after several tests on the data traffic for the cars plying the roads using the normal traffic light. The average number of cars on each side of the road per minute is given in tables below.
Simulation result on average number of the cars on the roads after the first test running using genetic algorithm Intelligent Traffic Light software is as shown in table 2 below.

Table 2: Simulation Result after test 1

\begin{tabular}{|c|c|}
\hline No. Cars on Road A per minute & 85 \\
\hline No. Cars on Road B per minute & 150 \\
& \\
\hline
\end{tabular}


Simulation result on average number of the cars on the roads after the second test running using genetic algorithm Intelligent Traffic Light software is as shown in table 3 below.

Table 3: Simulation Result after Test 2

\begin{tabular}{|l|c|}
\hline No. Cars on Road A per minute & 25 \\
\hline No. Cars on Road B per minute & 60 \\
\hline
\end{tabular}

Simulation result on average number of the cars on the roads after the third test running using genetic algorithm Intelligent Traffic Light software is as shown in table 4 below.
The experimental analysis of simulation results is summarized in table 6 and figure 8

Table 4: Simulation Result after Test 3

\begin{tabular}{|c|c|}
\hline No. Cars on Road A per minute & 40 \\
\hline No. Cars on Road B per minute & 46 \\
\hline
\end{tabular}

The average number the simulation traffic data collected from the tables above are given table 5 .

Table 5: Average of the Test Results

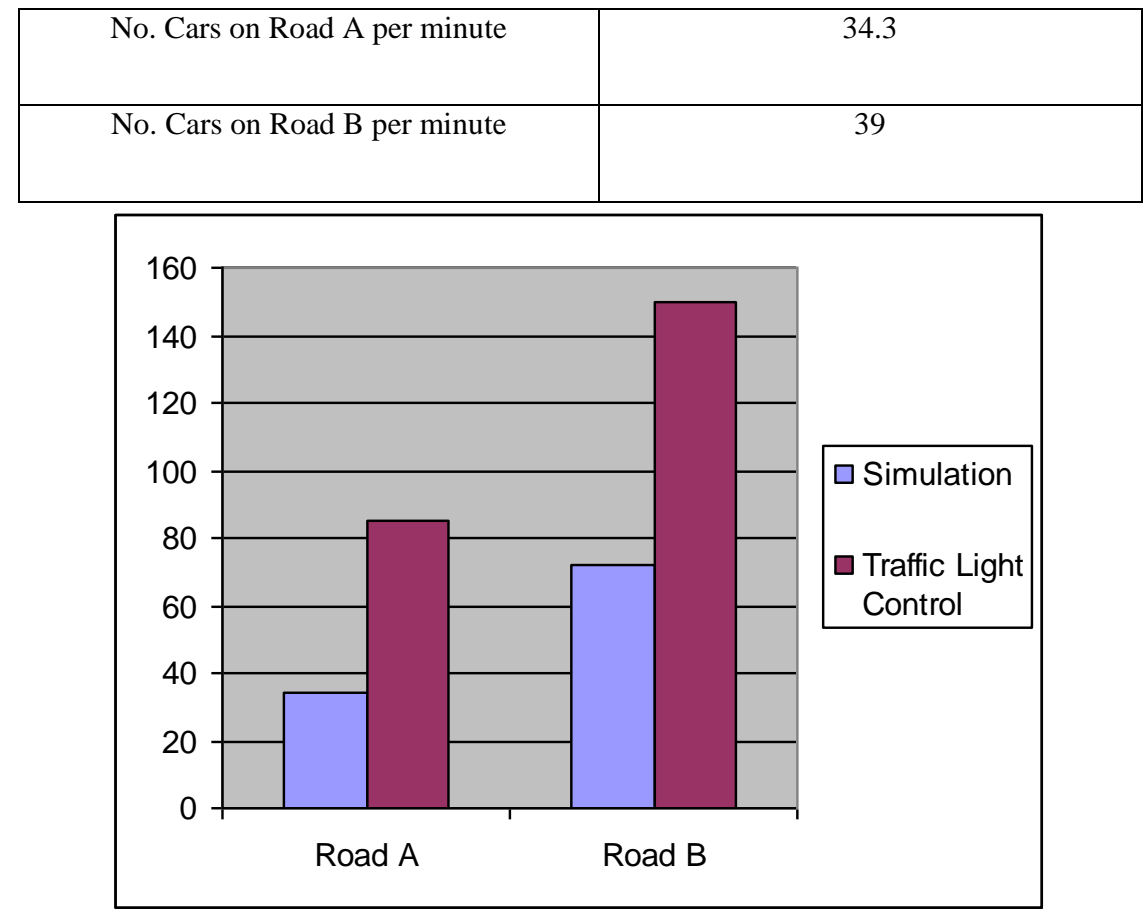

Fig.7: Graphical Representation of the Simulation Results

Table 6: Experimental Analysis

\begin{tabular}{|c|c|c|c|c|}
\hline & $\begin{array}{c}\text { Road A Traffic } \\
\text { Interval (Secs) }\end{array}$ & $\begin{array}{c}\text { Road B Traffic } \\
\text { Interval(Secs) }\end{array}$ & $\begin{array}{c}\text { No Cars on Road } \\
\text { A after } \\
\text { simulation }\end{array}$ & $\begin{array}{c}\text { No Cars on Road } \\
\text { B after } \\
\text { simulation }\end{array}$ \\
\hline Test 1 & 30 & 30 & 29 & 30 \\
\hline
\end{tabular}




\begin{tabular}{|c|c|c|c|c|}
\hline Test 2 & 25 & 25 & 35 & 34 \\
\hline Test 3 & 15 & 15 & 39 & 38 \\
\hline Test 4 & 10 & 10 & 42 & 45 \\
\hline
\end{tabular}

After the test, the following results showing the number of cars left on the road for each time interval of the traffic light in the simulation is shown in table 6 .

After several runs of simulation test as shown in table 1 to 5 above, the results show that the proposed Traffic intelligent system which is based on genetic algorithm show more promising result in terms of traffic prediction and better functionality in finding the best fit to be off peak period, where more nature of the road is being active to reduce traffic jam. Result show that the off peak period are more than effective than the peak period. Because of the vehicle composition rating is $>40 \%$,

Where that of peak period was $>50 \%$, which is not fit for the urban area. The graphical representation of the traffic data comparing the number of cars left on the road after a minute using both the traffic light control and the intelligent simulation software is represented in figure 7 above. It can be observe from the outcome of the test that the simulation had the least number of cars on the road after a minute showing that the use of genetic algorithm for traffic light control system will help to reduce the traffic congestion on the roads by reducing the number of cars left after the traffic light stop. From the graph it can be seen that the value with the lowest number of cars is the test 1 where we have the highest traffic control time interval of 30 seconds. This was the best case test scenario for reduction of traffic.

The worst case test scenario was the test 4 which has the highest amount of cars left on the road with a traffic light control interval of 10 seconds.

\section{CONCLUSION AND FURTHER WORK}

In conclusion, the Traffic Light Intelligent System which is based on the genetic Algorithm holds great promises as a future Traffic light monitoring model if properly planned. the data obtained from the simulations done using the proposed system indicates better performance of the system. Thus, modeling intelligent system is suitable for simulating the traffic lights at intersections. Hence, accurate simulation of traffic is close to reality.

Further work will be on integration of the Traffic Light Intelligent model into real time hardware, as the system has not been integrated into real time hardware so it may not be obvious if it will perform as expected and finally, the output is not symbolic i.e. cannot be interpreted as a mathematical

\section{REFERENCES}

[1] Goldberg,D. E. 2008. Genetic Algorithms in Search, Optimization, and Machine Learning. Upper Saddle River, NJ: Addison-Wesley.

[2] Haupt R. and G. Panchal. 2012. Classification and Optimization to Evaluate the Fitness of an Algorithm. Lap Academic Publisher, Germany, 2012.

[3] Gerla,M., et al. 2005, "Internet of vehicles: from intelligent grid to autonomous cars and vehicular clouds," in Proceedings of the IEEE World Forum on Internet of Things (WF-IoT '14), pp. 241-246.

[4] Abedinnia, H.2016, "New simple constructive heuristic algorithms for minimizing total flow-time in the permutation flowshop scheduling problem," Computers and Operations Research, vol. 74, pp. 165-174

[5] Branks, J.and Carson J. 2005. Discrete-event system simulation (4th Ed.). Upper Saddle River, NJ: Pearson Prentice Hall. ISBN 978-0-13-088702-3.

[6] Assad, A. A., 2002. Models for rail transportation. Transportation Research Part A: General Volume 14, Issue 3, 205-220

[7] Aldrich, C. 2003. Learning by Doing: A Comprehensive Guide to Simulations, Computer Games, and Pedagogy in e-Learning and Other Educational Experiences. San Francisco: Pfeifer - John Wiley \& Sons. ISBN 978-07879-7735-1.

[8] Ayad, M. 2009. Use of Genetic algorithm for Traffic Light and Pedestrian Crossing Control, (2009). pp.1-2.

[9] LiviuOana (2017). Use of Genetic Algorithms in Numerical Weather PredictionInternational Symposium on Symbolic and Numeric Algorithms for Scientific Computing (SYNASC), 78-84

[10] Peter, M. 1988; Search for articles Herbivores and plant 132: 869-883.

[11] Oppenheim, A. 1992. Questionnaire Design, Interviewing and Attitude Measurement, London, Pinter. Pp 303.

[12] Gaurav, K. and Pradeep, K. 2012. Impact of Agile Methodology on Software Development Process: International Journal of Computer Technology and Electronics Engineering (IJCTEE) Volume 2, Issue 4 